\title{
COMBINING VIRTUAL REALITY ENABLED SIMULATION WITH 3D SCANNING TECHNOLOGIES TOWARDS SMART MANUFACTURING
}

\author{
Windo Hutabarat \\ John Oyekan \\ Christopher Turner \\ Ashutosh Tiwari \\ Cranfield University \\ College Road \\ Cranfield \\ Bedford, MK43 0AL, UK
}

\author{
Neha Prajapat \\ Xiao-Peng Gan
}

GE Power

Newbold Road

Rugby, CV21 2NH, UK

\author{
Anthony Waller \\ Lanner Group \\ Forward House \\ 17 High St \\ Henley-in-Arden, B95 5AA, UK
}

\begin{abstract}
Recent introduction of low-cost 3D sensing and affordable immersive virtual reality have lowered the barriers for creating and maintaining 3D virtual worlds. In this paper, we propose a way to combine these technologies with discrete-event simulation to improve the use of simulation in decision making in manufacturing. This work will describe how feedback is possible from real world systems directly into a simulation model to guide smart behaviors. Technologies included in the research include feedback from RGBD images of shop floor motion and human interaction within full immersive virtual reality that includes the latest headset technologies.
\end{abstract}

\section{INTRODUCTION}

Within the space of a few short years we have seen the introduction of affordable 3D sensing through the Kinect sensor and its ilk along with affordable immersive virtual reality (VR) head-mounted displays (HMD). These technologies has significantly lowered the cost of creating and maintaining 3D virtual worlds. In this paper we will describe our attempt to leverage these technologies to help in the use of simulation, particularly discrete-event simulation (DES). We will limit our the scope of our discussion to manufacturing shop floor layouts.

Visualization is important for manufacturing simulation (Rohrer 2000), and the importance of VR visualization techniques to DES was recognised early on (Taylor and Robinson 2006). VR-enabled DES is viewed as a suitable tool to help address many manufacturing challenges (Dorozhkin et al. 2010) and accordingly a recent survey by Choi, Jung, and Noh (2015) found numerous implementations of VR with manufacturing simulation. The launch of affordable VR HMDs open up the possibility of immersive visualisation and creation of DES simulation on every workstation. Examples of their use in creating 
virtual worlds for gaming is not long in coming (Figure 1). Although these authoring approaches are still at their infancy, already we can see the possibilities that it can bring to DES modelling.
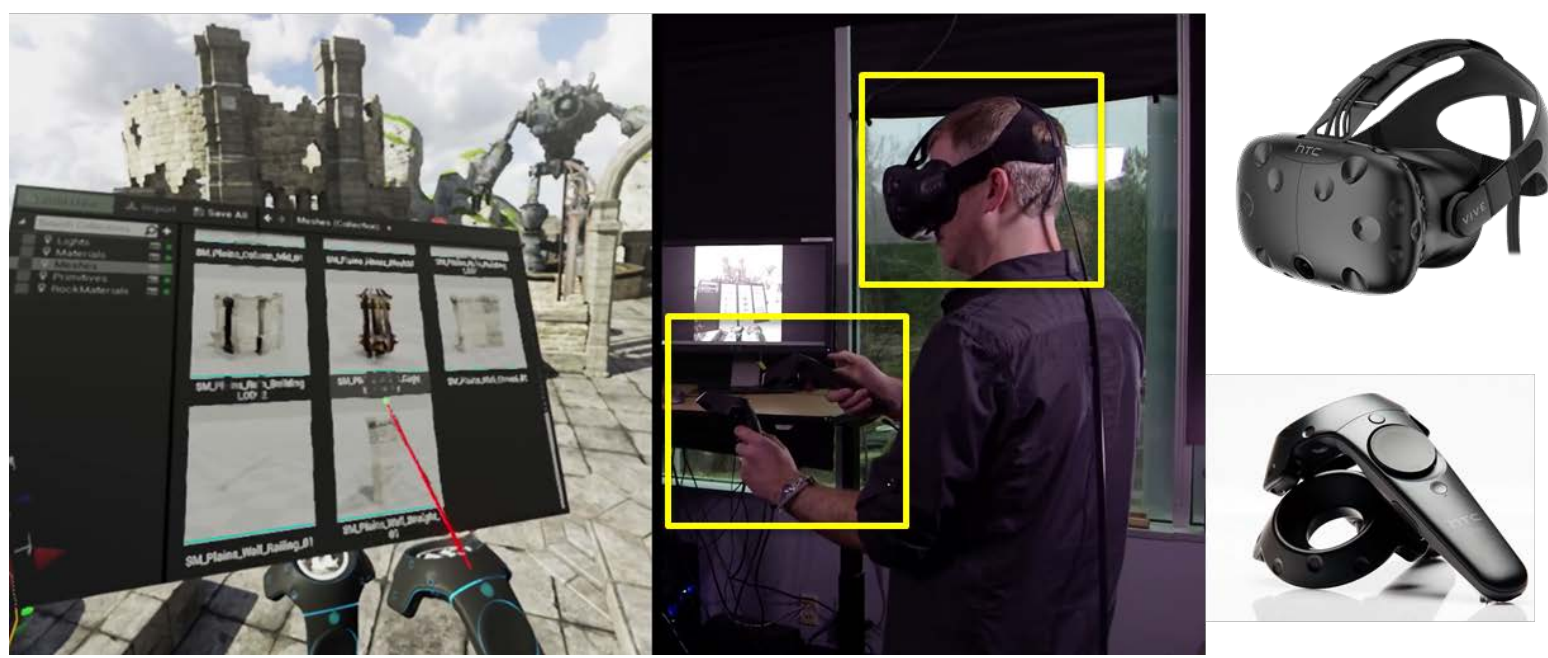

Figure 1: The new VR HMD devices are well supported by game authoring platforms, allowing VR world building in VR environments (Sweeney and Fricker 2016). Shown are HTC Vive HMD and its hand-held controllers.

The opportunity from recent developments in the use of low cost RGBD 3D sensors is to gain realtime data about events on the shop floor, which include humans actions and material and equipment movements. RGBD sensors such as Microsoft Kinect are integrated visible spectrum (termed RGB for Red-Green-Blue) and depth (D) sensors that provides color and depth data streams. In many implementations, they are paired with middleware that can, at negligible cost, robustly produce anonymous skeletal tracking data from depth data. Prabhu et al. (2015) has shown that these capabilities makes it possible to extract re-usable details on manufacturing tasks (Figure 2).

Concurrently, there is growing realisation of the importance of data analytics and its compatibility with DES concepts. Nelson (2016) recognised that simulation's predictive power complements the interpolative power of machine learning, opening up the possibility of using simulation in concert with machine learning for extrapolation from big data. There is increasing attention for using wireless sensor networks to collect data on manufacturing operations. These sensors are often physically connected to machines in order to measure and keep track of material flows in the manufacturing plant (Suto et al. 2015). This approach is promising but misses out the human component that is highly influential to plant performance (Mason et al. 2005), which can be supplied by the RGBD system.

The Industry 4.0 initiative promises improvements in manufacturing environment through the use of cyber-connected sensors that collect 'big data' from machines and people at a detailed level. This opens up the possibility of new ways for understanding manufacturing processes as well as performing predictive analysis.

In this paper, we discuss progress towards integrating a DES with affordable VR HMD visualisation and data obtained from low-cost RGBD sensor. Firstly, we will describe our proposed architecture and how the new technological integration can bring benefits. Next, we will describe our progress in DES VR interfacing. Next, we will discuss our implementation of DES-RGBD interface. Finally we will close with some thoughts about our next steps. 


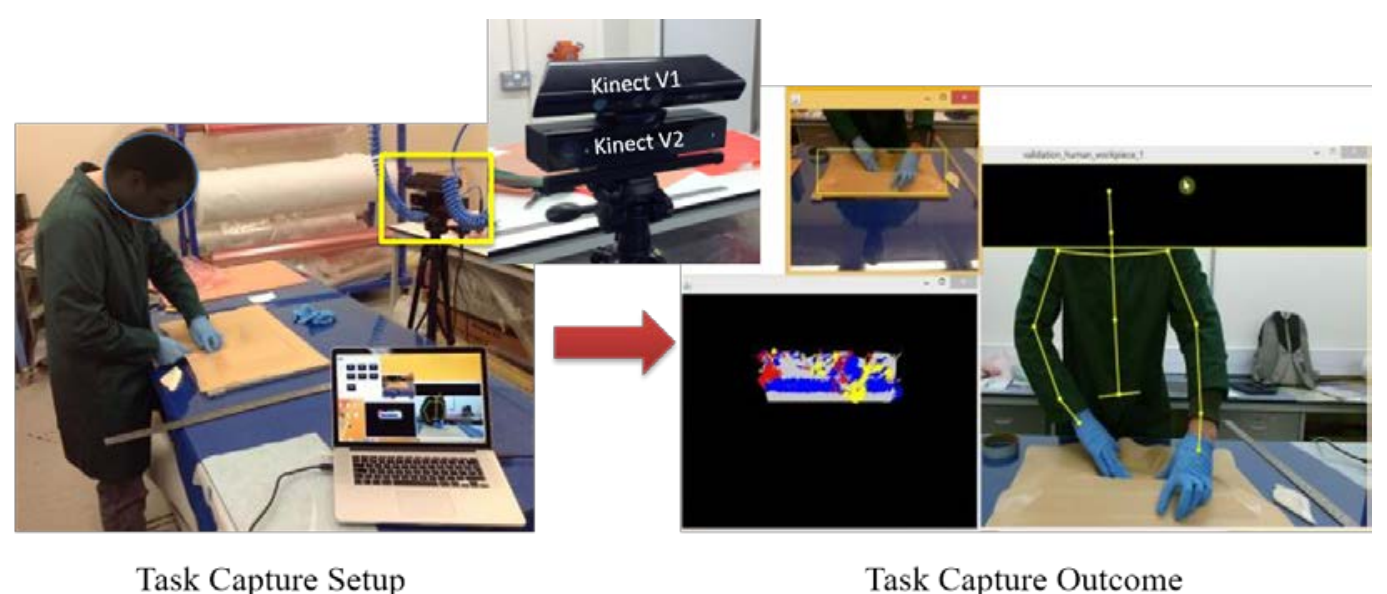

Figure 2: Using RGB-D sensors to capture manufacturing task. The worker is performing a composite lay-up task on a workbench. One sensor (Kinect v1) is dedicated to capturing the workpiece states while another sensor (Kinect v2) captures the worker skeleton movements (Prabhu et al. 2015).

\section{ENVISIONED SYSTEM INTEGRATION}

In this work the authors have two goals: to create an immersive interface for users of VR-DES, and to use RGB-D information from low-cost sensors in DES. By combining DES with VR and RGB-D sensing, the authors envision new affordances to emerge as depicted in Table 1. The rationale for each is explained in the following paragraphs.

Table 1: Pairwise affordances envisioned from the proposed integration of DES, VR technologies, and low-cost RGBD sensing.

\begin{tabular}{|r|l|l|}
\hline A/B & A to B affordances & B to A affordances \\
\hline DES/VR & $\begin{array}{l}\text { New methods to visualize the } \\
\text { complexity of shop floor behavior }\end{array}$ & Novel DES authoring approaches \\
\hline DES/RGBD & Automated machine vision setup & Automated update of events \\
\hline RGBD/VR & Real-time updates of workspace changes & Immersive visualization of workspaces \\
\hline
\end{tabular}

DES to VR affordance: The link between DES and VR seem well explored (Choi, Jung, and Noh 2015; Ghani, Monfared, and Harrison 2015) but the active research into big data visualization using immersive VR (Olshannikova et al. 2015) suggests the possibility of undiscovered methods for immersive visualization of simulation models. Nelson (2016) identified a need to gain insight into the complexity of shop floor behavior, for which novel immersive VR may provide imaginative new answers. Immersive visualization also provides a new medium for trying out innovative animation methods in ways akin to Zhong and Shirinzadeh (2008).

VR to DES affordance: DES VR models are mostly created on standard 2D screens. Traditional VR equipment are so scarce and costly that they are more suitable for occasional design reviews rather than for day-to-day high-intensity model creation tasks. With affordable and ubiquitous VR, we can explore new user interface approaches for authoring DES models, perhaps in similar vein to that shown in Figure 1. It is possible that there are undiscovered editing methods that make sense only in immersive VR.

DES to RGBD affordance: Setting up intelligent machine vision systems generally require a great deal of work. Calibration, context definition, and relevant scenarios need to be set up in such a way that vision algorithms can recognize and classify any unfolding scene. Accurate VR DES models already contain a great deal of information that can be leveraged to automate the setting up of an intelligent 
RGBD vision system. For instance, the scene geometry information alone should be sufficient to train RGBD vision systems to recognize objects (Lai, Bo, and Fox 2014). The real-time point clouds can then be used to detect discrepancies, as demonstrated by Nahangi et al. (2015) for assembly operations and Wang and Cho (2015) in construction.

RGBD to DES affordance: The approach proposed by Prabhu et al. (2015) provides detailed quantitative knowledge about manufacturing tasks and human actions. This information can be collected and then leveraged to update DES models, which may contain inaccurate assumptions. For instance, collected data about worker cycle times can be used to calibrate the workers' probability density functions (Mason et al. 2005).

RGBD to VR affordance: Point clouds obtained by laser scanners have been used to update VR manufacturing environments but they can only be done occasionally (Shellshear, Berlin, and Carlson 2015). Low cost RGBD sensors pointing at areas of interest can provide real-time updates of any changes in indoor workspaces (Henry et al. 2012), e.g. for providing alerts in real-time when collision points arise.

VR to RGBD affordance: Such a network of RGBD sensors creates a real-time VR image that can be directly visualized using VR HMDs, providing an additional means for verification of workspace states (Yang et al. 2011).

Finally, the full integration of DES, VR, and RGBD sensor open up tantalizing possibilities of creating a fully synced, up-to-date 'virtual factory' (Jain and Shao 2014; Yang et al. 2015) which will lend itself to the simulation analytics approach proposed by Nelson (2016).

Our work is still ongoing. Still to come is our RGBD/VR interfacing and the DES/VR/RGBD final integration. In the next sections we will report on our progress in DES/VR interface development and in implementing DES/RGBD integration.

\section{DISCRETE EVENT SIMULATION AND VIRTUAL REALITY}

The aim of our ongoing DES/VR work is to develop a two-way link between DES software and the VR visualization software. Our starting point was the VR version of Lanner Group's WITNESS DES software, which already share VR geometry through socket links with Virtalis' Visionary Render VR software. Our focus is on shop floor layout design, where the visualization interface should help decision makers visualize activities and material flows so that they can devise the best shop floor arrangement. The socket communication backbone was re-used between VR to DES to create two-way communication between the two software. In Oyekan et al. (2015) we discussed the challenge of interacting with DES through an Oculus Rift DK2 HMD immersive interface and presented an example where operational parameters of a simulated factory floor was modified through an immersive interface enabled by a VR HMD.

One of the identified challenge is the lack of knowledge on how to design user interfaces for immersive VR environments. Existing methods which was mostly designed for CAVE environments usually do not take into account the fact that when wearing immersive HMD users are completely cut off from their surroundings. This cutting off does enhance immersiveness but present unique challenges, e.g. the risk of motion sickness is greatly magnified. A consensus on the design of immersive virtual reality interfaces is emerging, mostly driven by game developers responding to the new VR technologies (Borrell 2016) and recently also from the mainstream developers attracted by the untapped potential of desktop immersive interfaces (Sundstrom 2015; Alger 2015). In our work, Souto Moure (2015) evaluates the applicability of ISO $9241-210: 2010$ on human-centred design for interactive systems and used it to devise a framework for immersive user interface for virtual reality DES (Figure 4). 


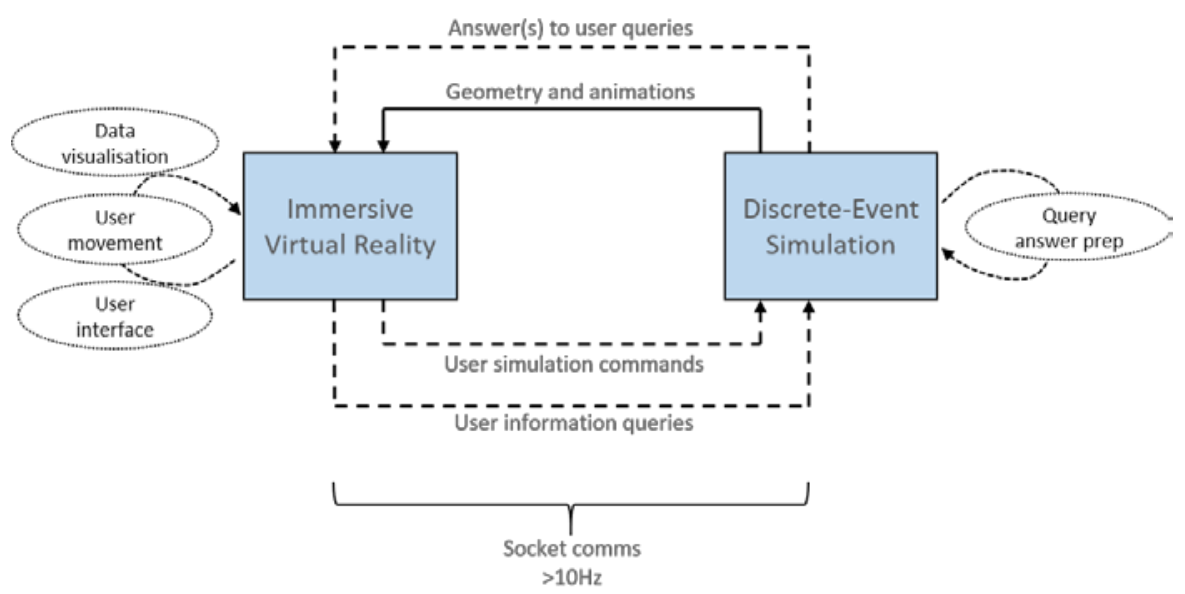

Figure 3: DES and VR interfacing implementation, adapted from (Oyekan et al. 2015).

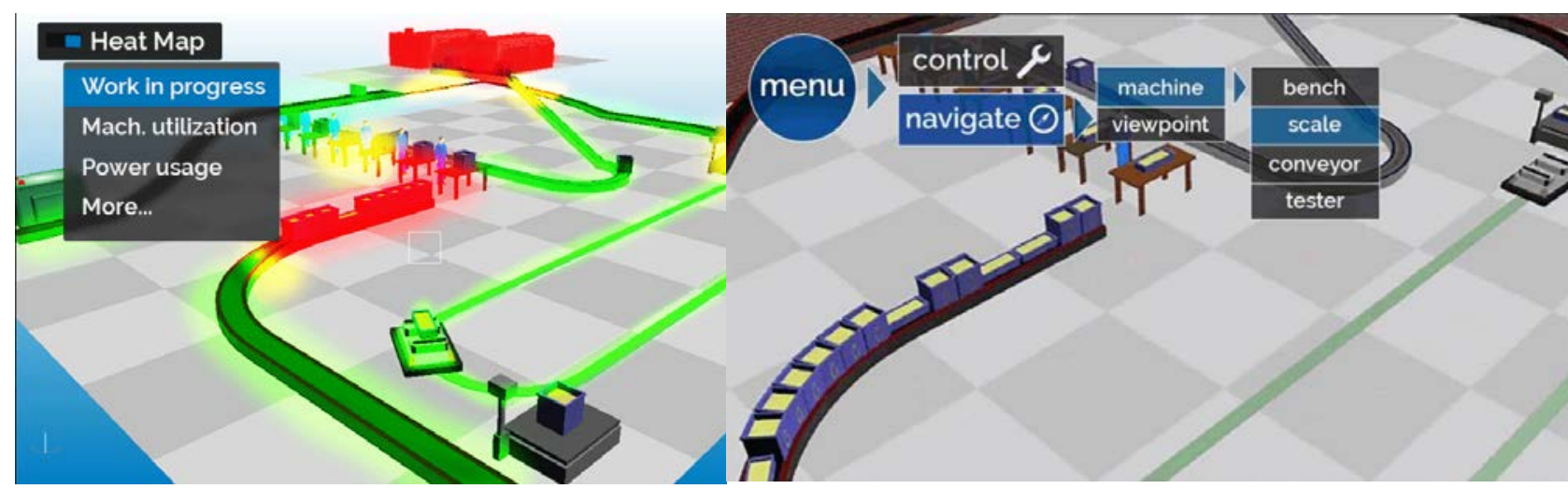

Figure 4: Examples of the resulting interface design from Souto Moure (2015) 's immersive VR interface framework for DES.

We also encounter practical challenges. The obvious solution when creating custom VR geometry for bespoke shop floors is to re-use or modify pre-existing CAD geometries. We discovered that inadequate attention towards geometrical complexity may create VR environments with too much detail, leading to unacceptable VR frame rate. Petti et al. (2016) found that when using high fidelity geometries, this frame rate reduction induces motion sickness which seriously hampered their immersive VR DES usability testing (Figure 5). The standard method to address this is by limiting the number of scene polygons. This is a routine task for game designers and their toolset; however, it is not a task that is well supported by CAD authoring tools. After substantial trial-and-error, this issue was mitigated by using a set of geometry conversion software to sequentially process the CAD geometries with close control on polygon count.

Our current focus is to develop ways in which users can maximize their productivity while in the immersive environment. We are developing representative use cases, devising and testing immersive VR DES interface for these cases, which will allow us to augment Souto Moure's work (2015) with new guidelines.

\section{DISCRETE EVENT SIMULATION AND RGBD IMAGING}

The aim of our ongoing DES/RGBD work is to achieve (i) data collection of a manufacturing process through RGBD sensing and scene understanding of the observed manufacturing environment, (ii) utilization of machine learning techniques to use simulation information for self-calibrating the RGBD 
system, and (iii) develop automated simulation analytics that are triggered by discrepancies in task execution or changes in the manufacturing environment. The RGBD system will be used to collect data from the shop floor in order to update a DES model of the shop floor processes. The model will be kept up to date with data from the shop floor so that it does not suffer from model ageing caused by worker non-compliance, part and material flow changes, environment and machine deviations, or model omissions. Furthermore, the inbuilt human motion capture technology in many RGBD sensors ensures that the effects of human actions on material flow in the factory can be captured as well. A top level data flow diagram of the envisioned system is depicted in Figure 6.
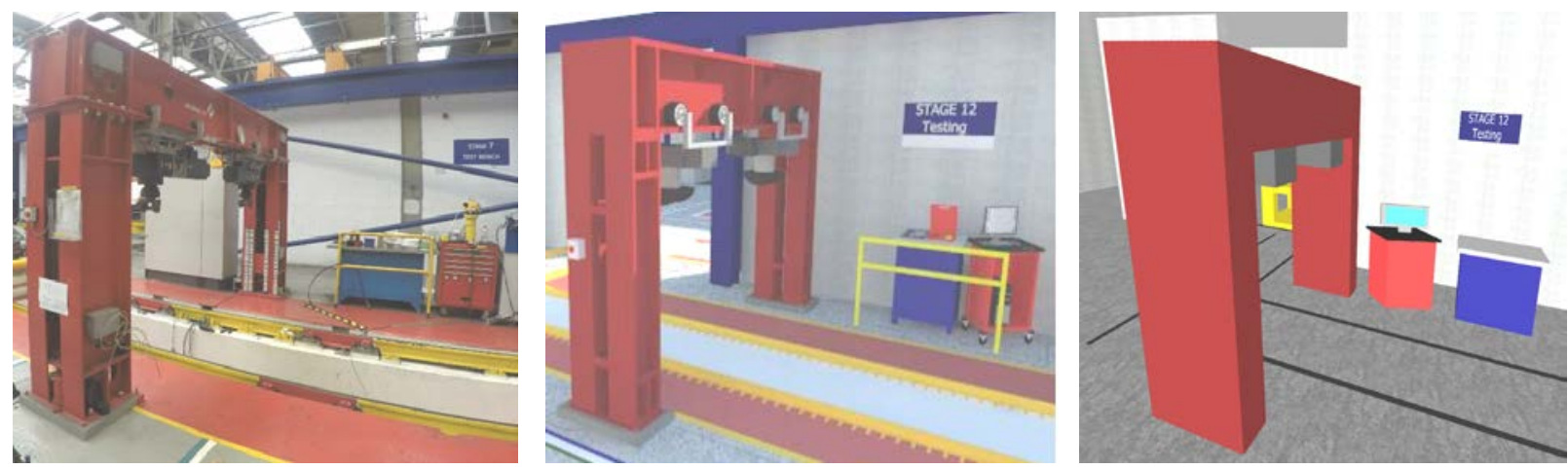

Figure 5: Actual shop floor and its immersive 3D models in high fidelity (middle) and low fidelity (right) (Petti et al. 2016) implemented in WITNESS 14 and viewed through Visionary Render.

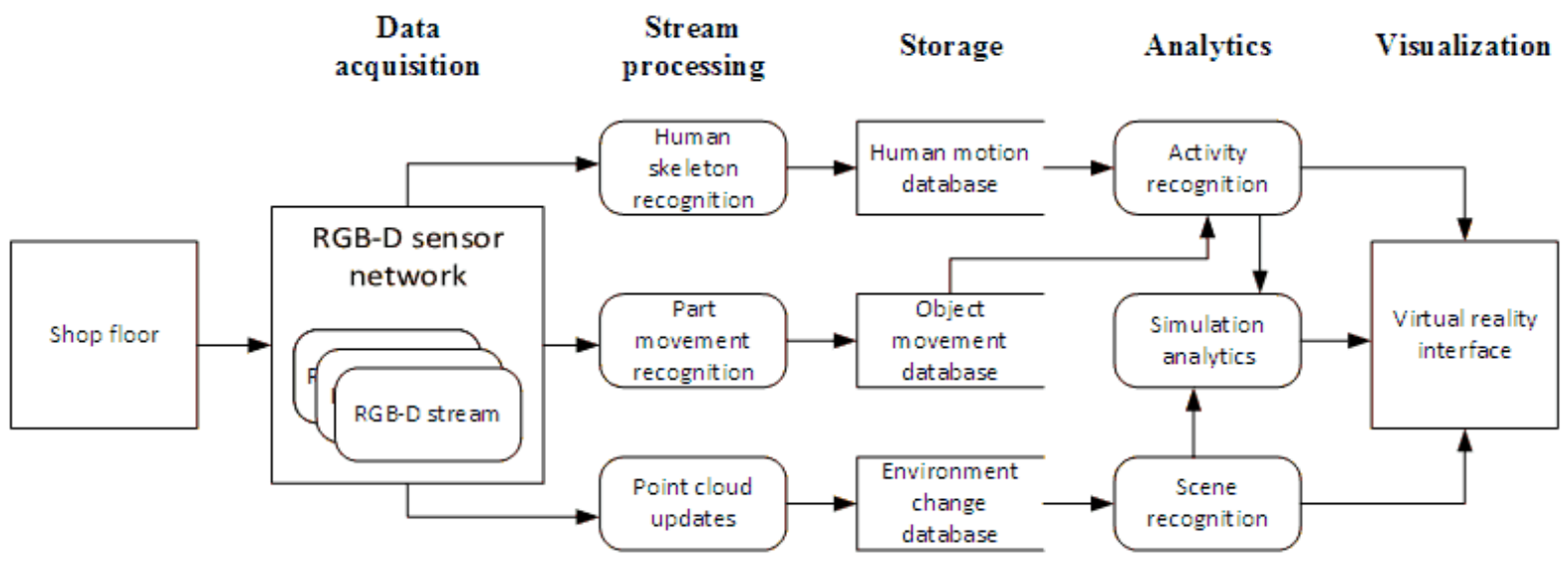

Figure 6: Data flow diagram for the envisioned RGBD system.

Our work is inspired by the advances in intelligent video analysis (IVA). Automated RGB video annotation is a well-established topic within computer vision and includes relevant challenges such as scene recognition, event detection and action recognition. It was found that RGBD sensors along with the skeleton detection allows robust activity recognition of human workers (Aggarwal and Xia 2014).

Automatic utilisation of depth sensor data on shop floors has been performed by Shellshear, Berlin, and Carlson (2015), whereby periodic laser scans generate factory layout dimensions, allowing automatic detection of emerging collision risks due to environmental changes or due to changes in part/product size. Similarly, Nahangi et al. (2015) analysed point cloud generated by laser scanners to provide autonomous 3D discrepancy feedback when fitting steel structures during construction. RGBD sensors cannot compete 
with laser scans in terms of absolute geometrical accuracy. However, the low cost and low computing requirements open up the possibility of setting up a network of RGBD sensor throughout the shop floor. This network can potentially perform full real-time tracking of human workers, mobile robots, parts, and products (Almazan and Jones 2013; Regazzoni, de Vecchi, and Rizzi 2014). In response to concerns about the difficulty of setting up and configuring a network of RGBD sensor, Munera et al. (2015) has demonstrated a technique whereby a RGBD sensor system can automatically adapt its usage of resources to ensure quality of detection.

In the next section, we will describe our progress towards automatically adapting the RGBD sensor to detect changes in material flow path.

\section{DISCRETE EVENT SIMULATION AND RGBD IMAGING CONCEPT DEMOSTRATION}

To demonstrate our approach, we use a Fischertechnik 'Indexed Line with Two Machining Stations' kit (art. 51664) as a substitute of an actual shop floor process. The Fischertechnik line processes a set of pucks which represent manufacturing workpieces. The simple system consisted of input and output buffers, conveyor belts, a spacing area, and two machining stations.

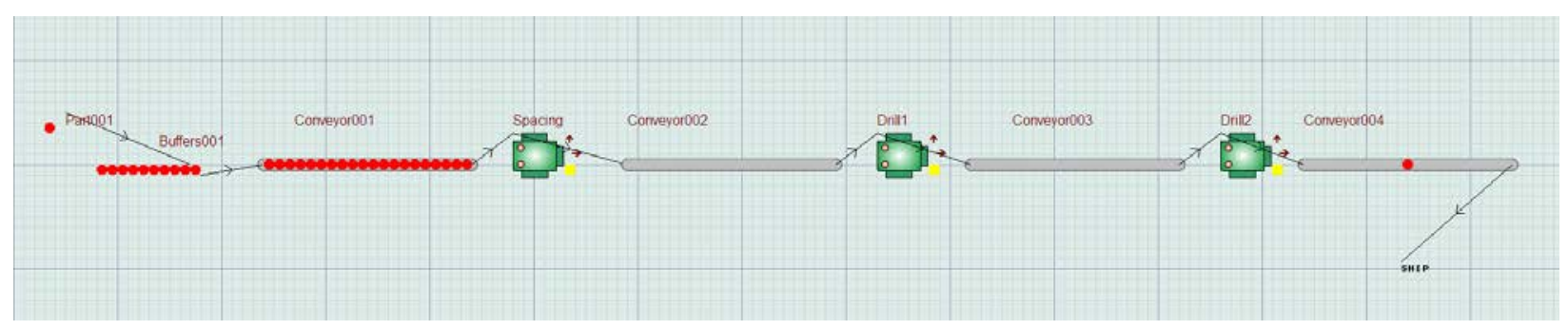

Figure 7: Simulation of a simple manufacturing line performed in WITNESS 14.

We start by defining a DES simulation model (Figure 7) of the Fischertechnik line in Figure 8. Both the DES model and the physical line are programmed deterministically. However, the rate of movement of the puck in the physical line is made highly variable by changing the geometry of the puck with adhesives. This creates variability which we hope to detect using the RGBD system and then feedback to update the DES model in the future.

A Kinect sensor was used to analyse a stream of RGBD data for the purposes of extracting material flow information. Two approaches of information extraction were investigated in this paper. Firstly we will describe our detection of material flow data from the shop floor carried out using 'flow detection' approach, where detection 'hot spots' are pre-defined on the sensors field of vision. Next, we demonstrate our 'change detection' approach, where the RGBD system can automatically adapt the hot spots to account for changes on material flow path. The methods implemented mostly rely on RGB signals, but it can be readily adapted to full RGBD data.

\subsection{Flow Detection Approach}

In this flow detection approach, hot spots were defined in the environment by dividing the observed space $O \in R^{2}$ into a grid of observable cells $\left\{o_{11}, o_{12}, \ldots \ldots . o_{N N}\right\}$ with resolution 10 by 10 pixels. Each cell is assigned a 'virtual photoreceptor' that is made up of two outputs: $\mathrm{P}(\mathrm{t})$, a function of the illumination at time $t$, and $H(t)$, a function of the previous illumination at time $t-T$.

$$
\begin{aligned}
& P(t)=k \ln \frac{E(t)}{E_{0}} \\
& H(t)=k \ln \frac{E(t-T)}{E_{0}}
\end{aligned}
$$


Hutabarat, Oyekan, Turner, Tiwari, Prajapat, Gan, and Waller

$$
f(x)=\left\{\begin{array}{c}
\text { Event, } P(t)-H(t)>\theta \\
\text { No Event, } P(t)-H(t)<\theta
\end{array}\right.
$$

Where $E(t)$ is the illumination at time $t, E(t-T)$ is the illumination at the time $(t-T)$ and is therefore a measure of illumination history while $\mathrm{K}$ and $\mathrm{E}_{0}$ are constants that define the photoreceptor sensitivity. In this work, $\mathrm{K}=1, \mathrm{E}_{0}=1$. A threshold scheme was used according to Equation 3 to define the occurrence of an event.

A priori virtual digital map of the region of interest was used in the data collecting software to associate detected event/state changes with the correct position of the puck through the assembly line. However, this naïve implementation will not always work. Illumination changes caused by lighting or object state changes e.g. assembly line tracks and lightning noise will cause detection failures, whereby objects appear to move between stations in random order.

In order to solve this challenge, a Markov chain (Figure 9) representative of the sequence of assembly line state changes was constructed and filled up with appropriate weight values. These values were determined empirically using knowledge of state changes in the DES model and the actual physical line.

This approach resulted in the filtering out ambiguous event state signals and the ability to keep track of a puck through a scaled down assembly line (Figure 8).
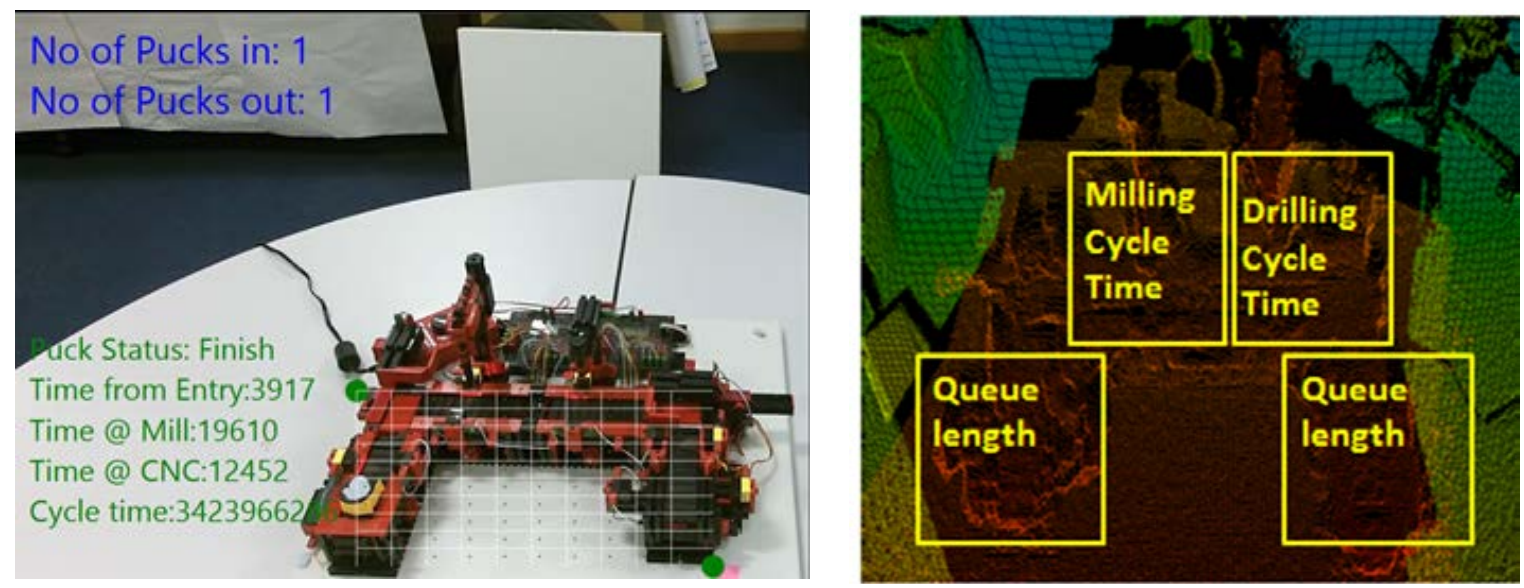

Figure 8: A view from the Kinect sensor on the Fischertechnik model of a machining line used as a development test bench: RGB image showing the pre-determined 'hot-spots' for material flow tracking (left) and the depth image obtained by the sensor (right).

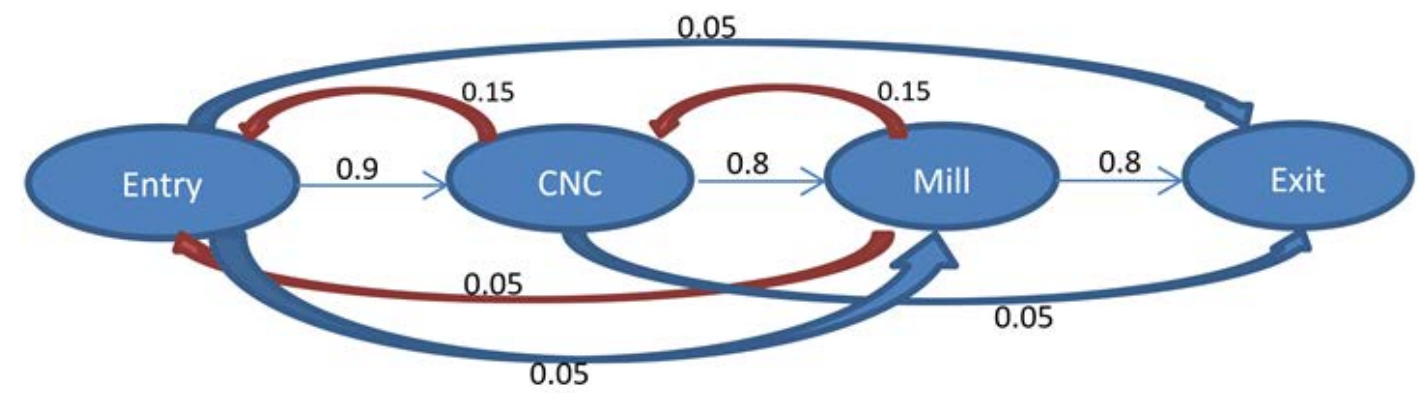

Figure 9: Empirically determined Markovian chain for reduction of false state changes. 


\subsection{Change Detection Approach}

The limitation of the flow detection approach is that the hot spots or interest points (green dots in Figure 8) need to be manually defined and different assembly layouts will need their virtual digital maps manually constructed. Furthermore, changes in the camera position and/or line position will lead to, at best, inaccurate mapping of puck state changes and will result in wrong data being created.

To mitigate these challenges, a pattern detection through k-means clustering was used following these steps:

1. Object detection: Through the use of a color thresholding and morphology scheme, the puck was separated from the rest of the scene. This enables the puck to be tracked as it progresses through the assembly line.

2. Data ingestion: time-stamped puck location data was stored in an expanding array.

3. Through the use of time threshold K-means clustering, the ingested data was divided into static and dynamic clusters. The time stamps from these clusters were then extracted in order to derive the time spent at each cluster (Figure 10, left).
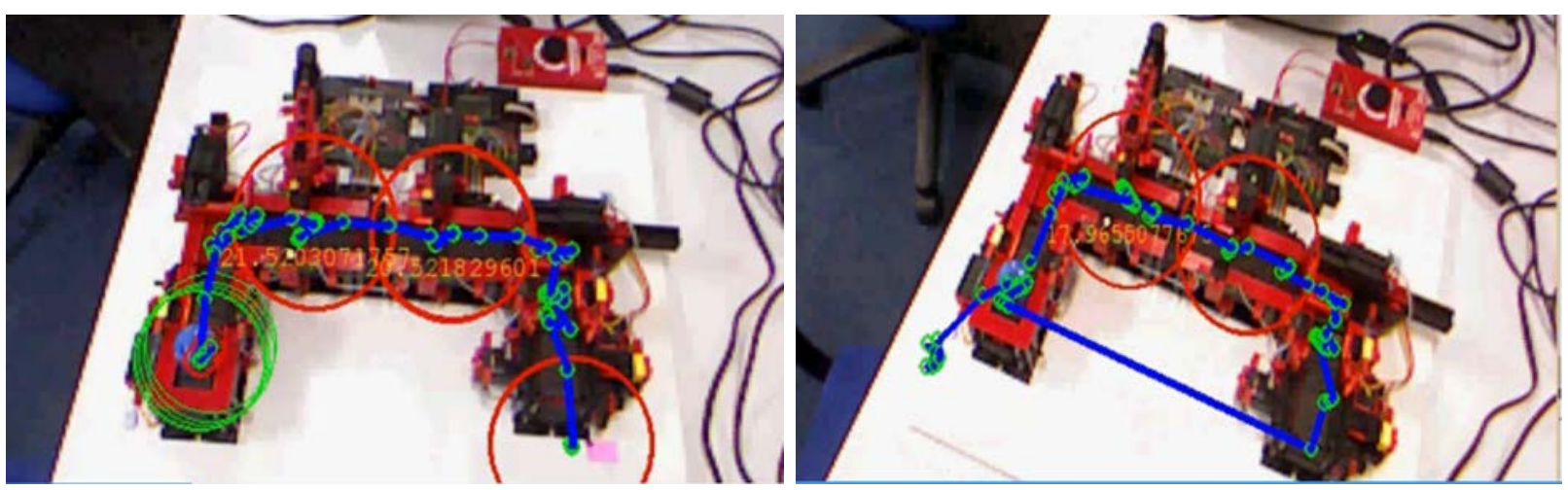

Figure 10: Detecting salient points of interest through the use of a time threshold k-means pattern detection algorithm: original orientation (left) and modified orientation (right).

Using the assumption that static clusters are either work stations or buffers, the above scheme made it possible to detect salient points of interest in the environment corresponding to the aforementioned locations. Furthermore, due to object recognition and tracking, it was possible to use one cycle of material flow through the system to detect these points of interest. As shown in Figure 10b, the result is an RGBD detection system that is less immune to environmental disturbances.

\subsection{Integration of Flow Detection and Change Detection Approaches}

Currently, the initial Markov chain filter is determined empirically for the flow detection approach. In future, this can be automatically extracted from the DES simulation model thereby putting into practice the automatic sensor setup afforded by the DES to RGBD link as discussed in Section 2. The change detection approach will be run regularly to maintain a robust virtual digital map of the assembly line. This map includes the detection hot spots essential to the flow detection scheme. Once the RGBD system is confident about the map and the Markov chain values, the flow detection approach will be used to collect real time data from the shop floor to update the DES model. After every data update, the DES model can be run to discover possible performance shortfalls, which can then be used to alert the user. This will put into practice the automatic updating of events afforded by the RGBD to DES link as stated in Section 2. 
Hutabarat, Oyekan, Turner, Tiwari, Prajapat, Gan, and Waller

\section{CONCLUSION AND FUTURE WORKS}

Our work so far has given us no reason to doubt the potential in the use of new 3D virtual world technologies to improve the use of DES. Immersive VR technology open up the possibility of new ways to interact with DES simulation, possibly allowing non-traditional DES to interact with DES models, manipulate the model using VR representations, and take advantage of insights created by DES from new visualisation approaches. Furthermore, we have demonstrated that DES to RGBD link affords automatic setup of RGBD sensing and automated update of events to the underlying DES simulation model. The advantage of our proposed scheme is that during setup, only the DES model of the facility needs to be constructed. The envisioned algorithms will be well-positioned to use for smart usage of data collected from the shop floor, putting us one step closer to a smart manufacturing system.

\section{ACKNOWLEDGMENTS}

The authors acknowledged the support of Innovate UK and EPSRC, provided through grant number EP/M506813/1 "Towards Zero Prototyping of Factory Layouts and Operations Using Novel Gaming and Immersive Technologies". All data supporting this study are provided as supplementary information accompanying this paper and can be accessed by contacting researchdata@cranfield.ac.uk.

\section{REFERENCES}

Aggarwal, J. K., and L. Xia. 2014. "Human Activity Recognition from 3D Data: A Review." Pattern Recognition Letters, Celebrating the life and work of Maria Petrou, 48 (October): 70-80. doi:10.1016/j.patrec.2014.04.011.

Alger, M. 2015. "Visual Design Methods for Virtual Reality." Ravensbourne. http://aperturesciencellc.com/vr/VisualDesignMethodsforVR_MikeAlger.pdf.

Almazan, E. J., and G. A. Jones. 2013. "Tracking People across Multiple Non-Overlapping RGB-D Sensors." In 2013 IEEE Conference on Computer Vision and Pattern Recognition, 831-37.

Borrell, A. 2016. "Introducing the Oculus Sample Framework for Unity 5." Oculus Developers Blog. March. https://developer.oculus.com/blog/introducing-the-oculus-sample-framework-for-unity-5/.

Choi, S., K. Jung, and S. D. Noh. 2015. "Virtual Reality Applications in Manufacturing Industries: Past Research, Present Findings, and Future Directions." Concurrent Engineering 23 (1): 40-63. doi:10.1177/1063293X14568814.

Dorozhkin, D. V., J. M. Vance, G. D. Rehn, and M. Lemessi. 2010. "Coupling of Interactive Manufacturing Operations Simulation and Immersive Virtual Reality." Virtual Reality 16 (1): 15-23. doi:10.1007/s10055-010-0165-7.

Ghani, U., R. Monfared, and R. Harrison. 2015. "Integration Approach to Virtual-Driven Discrete Event Simulation for Manufacturing Systems." International Journal of Computer Integrated Manufacturing 28 (8): 844-60. doi:10.1080/0951192X.2014.924159.

Henry, P., M. Krainin, E. Herbst, X. Ren, and D. Fox. 2012. "RGB-D Mapping: Using Kinect-Style Depth Cameras for Dense 3D Modeling of Indoor Environments." The International Journal of Robotics Research 31 (5): 647-63. doi:10.1177/0278364911434148.

Jain, S., and G. Shao. 2014. "Virtual Factory Revisited for Manufacturing Data Analytics." In Proceedings of the 2014 Winter Simulation Conference, edited by A. Tolk, S. Y. Diallo, I. O. Rhyzhov, L. Yilmaz, S. Buckley, and J. A. Miller. 887-98. Piscataway, New Jersey: Institute of Electrical and Electronics Engineers, Inc.

Lai, K., L. Bo, and D. Fox. 2014. "Unsupervised Feature Learning for 3D Scene Labeling." In 2014 IEEE International Conference on Robotics and Automation (ICRA), 3050-57.

Mason, S., T. Baines, J. M. Kay, and J. Ladbrook. 2005. "Improving the Design Process for Factories: Modeling Human Performance Variation.” Journal of Manufacturing Systems 24 (1): 47-54. 
Munera, E., J.-L. Poza-Lujan, J.-L. Posadas-Yagüe, J.-E. Simó-Ten, and J. F. Blanes Noguera. 2015. "Dynamic Reconfiguration of a RGBD Sensor Based on QoS and QoC Requirements in Distributed Systems." Sensors 15 (8): 18080-101.

Nahangi, M., J. Yeung, C. T. Haas, S. Walbridge, and J. West. 2015. "Automated Assembly Discrepancy Feedback Using 3D Imaging and Forward Kinematics." Automation in Construction 56 (August): 3646. doi:10.1016/j.autcon.2015.04.005.

Nelson, B. L. 2016. “'Some Tactical Problems in Digital Simulation' for the Next 10 Years.” Journal of Simulation 10 (1): 2-11. doi:10.1057/jos.2015.22.

Olshannikova, E., A. Ometov, Y. Koucheryavy, and T. Olsson. 2015. "Visualizing Big Data with Augmented and Virtual Reality: Challenges and Research Agenda." Journal of Big Data 2 (1): 1. doi:10.1186/s40537-015-0031-2.

Oyekan, J., W. Hutabarat, C. Turner, A. Tiwari, N. Prajapat, N. Ince, X.-P. Gan, and T. Waller. 2015. “A 3D Immersive Discrete Event Simulator for Enabling Prototyping of Factory Layouts." Procedia CIRP, Proceedings of the 4th International Conference on Through-life Engineering Services, 38: 6367. doi:10.1016/j.procir.2015.08.043.

Petti, A., W. Hutabarat, J. Oyekan, C. J. Turner, A. Tiwari, N. Prajapat, and X.-P. Gan. 2016. "Impact of Model Fidelity in Factory Layout Assessment Using Immersive Discrete Event Simulation." In Proceedings of the Operational Research Society Simulation Workshop 2016.

Prabhu, V. A., A. Tiwari, W. Hutabarat, and C. Turner. 2015. "Capture, Digitisation and Segmentation of Human-Workpiece Interactions in a Manual Assembly Operation Using Kinect ${ }^{\mathrm{TM}}$." International Journal of Design Engineering 6 (1): 61-85.

Regazzoni, D., G. de Vecchi, and C. Rizzi. 2014. "RGB Cams vs RGB-D Sensors: Low Cost Motion Capture Technologies Performances and Limitations." Journal of Manufacturing Systems 33 (4): 719-28. doi:10.1016/j.jmsy.2014.07.011.

Rohrer, M. W. 2000. "Seeing Is Believing: The Importance of Visualization in Manufacturing Simulation." In Proceedings of the 2000 Winter Simulation Conference, edited by B. Johansson, S. Jain, J. Montoya-Torres, J. Hugan, and E. Yücesan, 1211-1216. Piscataway, New Jersey: Institute of Electrical and Electronics Engineers, Inc.

Shellshear, E., R. Berlin, and J. S. Carlson. 2015. "Maximizing Smart Factory Systems by Incrementally Updating Point Clouds." IEEE Computer Graphics and Applications 35 (2): 62-69.

Souto Moure, A.. 2015. "Exploration of Human-Computer Interaction for Immersive Virtual Reality in Discrete Event Simulation." MSc Thesis, Cranfield University.

Sundstrom, M. 2015. "How to Design for Virtual Reality - Backchannel." Medium. April 16. https://backchannel.com/immersive-design-76499204d5f6.

Suto, K., H. Nishiyama, N. Kato, and C. W. Huang. 2015. "An Energy-Efficient and Delay-Aware Wireless Computing System for Industrial Wireless Sensor Networks.” IEEE Access 3: 1026-35..

Sweeney, T., and M. Fricker. 2016. Building VR in VR with Unreal Engine 4 - Early Preview. Epic Games. https://www.youtube.com/watch?v=JKO9fEjNiio.

Taylor, S. J. E., and S. Robinson. 2006. "So Where to next? A Survey of the Future for Discrete-Event Simulation." Journal of Simulation 1 (1): 1-6.

Wang, C., and Y. K. Cho. 2015. "Smart Scanning and near Real-Time 3D Surface Modeling of Dynamic Construction Equipment from a Point Cloud." Automation in Construction 49 (January): 239-49. doi:10.1016/j.autcon.2014.06.003.

Yang, X., E. Deines, and J. C. Aurich. 2011. "Visualization in Human-Centered Virtual Factories." In OASIcs-OpenAccess Series in Informatics, vol. 19. Schloss Dagstuhl-Leibniz-Zentrum für Informatik doi:10.4230/OASIcs.VLUDS.2010.111.

Yang, X., R. C. Malak, C. Lauer, C. Weidig, H. Hagen, B. Hamann, J. C. Aurich, and O. Kreylos. 2015. "Manufacturing System Design with Virtual Factory Tools." International Journal of Computer Integrated Manufacturing 28 (1): 25-40. 
Zhong, Y., and B. Shirinzadeh. 2008. "Virtual Factory for Manufacturing Process Visualization." Complexity International 12.

\section{AUTHOR BIOGRAPHIES}

WINDO HUTABARAT is a Research Fellow at the Cranfield's Manufacturing Informatics Centre, where he pioneered the idea of applying the innovations from the gaming sector into manufacturing and services that resulted in multiple research and development projects funded by Innovate UK. His interest is in optimisation, simulation, and soft computing. His email address is w.hutabarat@cranfield.ac.uk.

JOHN OYEKAN is a Research Fellow in the Manufacturing Informatics Centre at Cranfield University. He has successfully worked on several EPSRC and Innovate UK projects in the areas of computational intelligence, robotics and human-machine interaction with various industrial partners such as RollsRoyce, Airbus, and Jaguar Land Rover. He is currently investigating the use of low cost gaming devices to improve productivity in manufacturing. His email address is j.o.oyekan@cranfield.ac.uk.

CHRIS TURNER is a Research Fellow and Project Manager within the Manufacturing and Materials Department at Cranfield University. He is research active in the fields of manufacturing simulation, manufacturing informatics, business process management and business process optimisation. He was involved in the successful completion of several UK research council funded projects with subjects ranging from business process optimisation to the simulation of product-service systems. His email address is c.j.turner@cranfield.ac.uk.

ASHUTOSH TIWARI is the Professor of Manufacturing Informatics and the Head of the Manufacturing Informatics Centre at Cranfield University which is internationally recognised for its research in optimisation of high-value manufacturing processes and digitisation of skill-intensive manufacturing. He has developed a strong research track record by leading, as PI, 8 EPSRC projects, 4 Innovate UK projects, 1 AMSCI project, and 3 projects funded by EPSRC Centres for Innovative Manufacturing. His email address is a.tiwari@cranfield.ac.uk.

NEHA PRAJAPAT is a research engineer in Alstom Power. Neha Prajapat received her BSc in Mathematics from the University of Leicester in 2012. She is currently a Statistical Methods Engineer in the Methodologies for Tools department at Alstom Power in Rugby. She is also pursuing her PhD in Manufacturing Process Optimization at Cranfield University. Her email address is neha.prajapat@power.alstom.com.

XIAO-PENG GAN is the Head of Optimization and Robust Design group within the Methodologies for Tools department at Alstom Power. He received his BSc in Mechanical Engineering from the University of Zhejiang, China. He completed his $\mathrm{PhD}$ in Mechanical Engineering at University of Bath. His research interests include: optimisation methodologies, statistical analysis, robust design, and thermal analysis. His email address is xiao-peng.gan@power.alstom.com.

ANTHONY WALLER received a BSc (Hons) in Statistics from the University of St. Andrews in 1981 and has worked in the field of Operational Research and particularly simulation ever since. He has worked on many diverse international assignments as a consultant and has helped develop simulation software in a product management role. He is currently the Customer Development Manager at the Lanner Group and is on the editorial board of the Journal of Simulation. His email address is twaller@lanner.com. 